\section{Roberto W. Romeo \\ Mario Floris \\ Francesco Veneri}

\title{
Area-scale landslide hazard and risk assessment
}

\section{Environ Geol (2006) DOI 10.1007/s00254-006-0294-1}

The online version of the original article can be found at http://dx.doi.org/10.1007/ s00254-006-0294-1

R. W. Romeo ( $\square)$

Research Centre on Natural Hazards, University of Urbino 'Carlo Bo', 'Sogesta' Scientific Campus, 61029 Urbino, Italy

E-mail: rwromeo@uniurb.it

Tel.: + 39-0722-304235

Fax: + 39-0722-304260

M. Floris

Research Centre on Geologic Risks,

University of Rome 'La Sapienza',

Doria-Pamphili Palace, Valmontone, Italy

F. Veneri

Institute of Engineering Geology,

University of Urbino 'Carlo Bo',

'Sogesta' Scientific Campus,

61029 Urbino, Italy

\section{Unfortunately, the following errors} occurred:

1. In Eq. 9 the right formula has ' $i$ ' and ' $T$ ' inverted (i.e., $i \geq I$ and not viceversa in both sides of the equation) and ' $f$ ' in the power of the exponential function is instead 'lambda' in greek-letter. The right equation should read:

$P[N(t) \geq 1, t \mid i \geq I]=1-\mathrm{e}^{-\lambda(i \geq I) t}$
2. In the References, the right journal citation of Genevois and Romeo 2003 is 'Geomech' and not 'Geomach'. It should read: Genevois R, Romeo RW (2003) Probability of failure occurrence and recurrence in rock slopes stability analysis. Int $\mathbf{J}$ Geomech ASCE 3:34-42 